\title{
The Influence of Author Degree Centrality and L-Index on Scientific Performance of Physical Education and Training Papers in China Based on the Perspective of Social Network Analysis
}

\author{
Bin Zhang $\mathbb{D}^{1},{ }^{1}$ Jian Wu $\mathbb{D}^{\mathbb{2}}{ }^{2}$ Qian Huang $\mathbb{D}^{1},{ }^{1}$ Yujiao Tan $\mathbb{D}^{1},{ }^{1}$ Lu Zhang $\mathbb{D}^{1},{ }^{1}$ Qian Zheng $\mathbb{D}^{1}$ \\ Yu Zhang $\mathbb{D},{ }^{1}$ Miao He $\mathbb{D}^{1},{ }^{1}$ and Wei Wang $\mathbb{D}^{1}$ \\ ${ }^{1}$ Xi'an Physical Education University, Xi'an 710065, China \\ ${ }^{2}$ Shanghai University of Sport, Shanghai 200438, China
}

Correspondence should be addressed to Jian Wu; wujian@sus.edu.cn

Received 4 May 2021; Revised 10 August 2021; Accepted 28 August 2021; Published 30 September 2021

Academic Editor: Fei Xiong

Copyright (c) 2021 Bin Zhang et al. This is an open access article distributed under the Creative Commons Attribution License, which permits unrestricted use, distribution, and reproduction in any medium, provided the original work is properly cited.

With the increasing frequency of scientific cooperation, research on the impact of scientific cooperation on scholars' scientific performance has become a hot topic in academic research. This paper mainly uses quantitative research methods such as bibliometrics and social network analysis to analyze the correlation between degree centrality, L-index, and scientific performance of the Chinese author of Physical Education and Training Science and draws the following conclusions: (1) degree centrality is positively correlated with the number of papers, average citations per paper, and H-index and (2) L-index is positively correlated with the number of papers, average citations per paper, and $\mathrm{H}$-index. Unlike previous studies on scientific collaboration networks and scholars' performance that focused on traditional network characteristics indicators, this study introduces a new network characteristics indicator, L-index, through which the degree of collaboration between authors and other important authors in the research field can be better assessed, which also provides a new direction for research related to research collaboration networks and scholars' performance; at the same time, it also provides a theoretical basis for subsequent research on the utility of scientific collaboration, a reference for the assessment of the research performance of scholars in other disciplines, and a theoretical reference for more scientific and comprehensive prediction of scholars' research performance in the future.

\section{Introduction}

With the development of society and the progress of science, scientific research becomes more and more complex. Price put forward the concept of "big science" for the first time, pointing out that modern scientific research is characterized by high investment intensity, multidisciplinary crossing, expensive experimental equipment, and complex research objectives [1]. Scientific cooperation is one of the effective ways to solve complex scientific research problems. Scientific cooperation refers to the knowledge production mode in which many people work together to complete the same research topic in the process of scientific research, and it is extremely important in the historical process of scientific development. Especially at a time when science is increasingly comprehensive and complex, scientific collaboration not only allows researchers to share their knowledge in different fields, their experience and resources, and complex and expensive research equipment but also stimulates innovative thinking and increases the efficiency of research. Therefore, scientific cooperation has become more and more frequent, and scientific research has gradually shifted from individual research to team research. Scientific research cooperation has gradually become an unstoppable development trend.

As scientific cooperation has become a common phenomenon in scientific research, research on the effectiveness of scientific cooperation has gradually become an important 
topic in the field of scientific cooperation. The effect of scientific cooperation on the scientific performance of scholars is an important aspect of the research on the effectiveness of scientific cooperation. As we all know, scientific performance is an important basis for the evaluation of scholars' academic levels. The higher the scientific performance of scholars, the richer their academic achievements and the greater the influence of their academic achievements. The evaluation of scholars' scientific performance not only can establish the correct value orientation of scientific research, promoting the sustainable and stable development of scientific research, but also can provide an objective basis for teacher recruitment, project application, government funding, and so on. By studying how scientific cooperation affects scholars' scientific performance, we can find out the factors affecting scholars' scientific performance and their internal correlation, so as to optimize the way of scientific cooperation and improve scholars' scientific performance.

As a discipline that encompasses both the social and natural science dimensions, the study of Physical Education and Training cannot be furthered and advanced without the reference significance brought by the author's scientific performance. This paper selects 10,386 papers in core journals of Physical Education and Training in the CSSCI database, and Python was used to visualize the correlation between degree centrality, L-index, and scientific performance. Stata was used to conduct linear regression on degree centrality, L-index, and scientific performance to discuss their correlation. Unlike previous studies on research collaboration networks and scholars' performance that focused on traditional network characteristics indicators, this study introduces a new network characteristics indicator, L-index, through which the degree of collaboration between authors and other important authors in the research field can be better assessed, which also provides a new direction for research related to research collaboration networks and scholars' performance; at the same time, it also provides a theoretical basis for subsequent research on the utility of scientific collaboration, a reference for the assessment of the research performance of scholars in other disciplines, and a theoretical reference for more scientific and comprehensive prediction of scholars' research performance in the future.

In addition to the introduction of the first part, this paper also includes the following sections. The second section introduces the theoretical basis of this research. The third section introduces the data source, the choice of variables, and the research method. The fourth section is the empirical analysis. Finally, the fifth section summarizes the conclusions and contributions of this study and points out the shortcomings in the study.

\section{Theoretical Basis}

2.1. The Scientific Cooperation. At present, academic circles have different definitions of scientific cooperation. Heffner believes that scientific research cooperation is a strong form of interaction between researchers in the process of scientific activities. They form an ideal model of scientific research cooperation through ideological and intellectual exchanges [2]. Ziman and Schmitt believe that scientific cooperation is generated after scientific development has reached a certain stage and gradually stabilized, during which scientific cooperation plays an extremely important role in improving the output of scientific knowledge [3]. Katz and Martin believe that scientific cooperation is the work of researchers together for the common purpose of creating new knowledge [4]. Zhao and Wen believe that scientific cooperation is a kind of scientific activity in which two or more researchers or organizations cooperate together to maximize scientific research output in order to complete a common research task, and its essence is resource sharing between collaborators [5]. Based on the concept of scientific cooperation put forward by different scholars, the author believes that scientific cooperation means that researchers focus on a specific scientific problem through resource sharing and cooperation and finally jointly complete scientific tasks with clear objectives and jointly publish scientific results. Among them, the collaborative publication of research results is the ultimate expression of scientific collaboration.

2.2. Scientific Performance. Scientific performance refers to that in the process of scientific activities, the research subjects, driven by subjective motivation, objective needs, and possible original basis for cooperation, carry out various forms of cooperation and produce various direct or indirect benefits, such as the direct benefits of papers, patents monographs, and so on. Indirect benefits include the improvement of scholars' knowledge, skills, reputation, and influence. There are qualitative evaluation and quantitative evaluation to evaluate the research performance of scholars. The qualitative evaluation mainly refers to peer review, which is also the traditional way to evaluate the scientific performance of scholars. But peer review has its drawbacks. First, it is less efficient because it requires experts to score each scientific achievement and give a written explanation. In addition, peer review is highly subjective, and the review results are easily affected by subjective factors such as experts' personal interests, majors, and emotions, which make it difficult to guarantee fairness and justice. Quantitative evaluation has been proposed and widely applied with the continuous growth of scientific achievements and the continuous development of scientometrics and bibliometrics. Quantitative evaluation evaluates the research performance of scholars through clear quantitative characteristics, which improves the efficiency and objectivity of the evaluation of the scientific performance of scholars. At present, the research on quantitative evaluation of scholars' scientific performance has been in-depth, and its evaluation indicators are also diverse, mainly including: number of papers, average citations per paper, impact factors, number of collaborators, $\mathrm{H}$-index, and so on. [6]. Integrating existing research, as a paper is the result of knowledge formed in the course of scientific activities, it is the most direct form of the output of scientific research. Its quantity and quality can, to a certain extent, relatively objectively reflect the contribution of scholars to the existing body of knowledge, and therefore, 
the main reference for evaluating the performance of scholars is their published papers [7-9].

In this paper, the number of papers, average citations per paper, and $\mathrm{H}$-index are selected as the evaluation indicators of scholars' scientific performance. The number of papers is the total number of papers published by each author. Although the number of papers does not fully represent the academic level and influence of a scholar, the production of high-quality papers is usually based on a certain number of publications. For example, Nobel laureates publish 13.1 papers per year on average when they are about 20 years old, while ordinary scholars publish 3.5 papers per year on average [10]. Average citations per paper refer to the ratio of the total number of citations per author to the number of papers. Average citations per paper are based on its quality. The more the average citations per paper receive, the higher the degree of "recognition" and the higher the quality of the paper is. Papers published by some authoritative scholars are cited as much as 200 times per year, while those published by ordinary scholars are less than 10 times [11]. H-index is a research performance evaluation index proposed by Hirsch that combines the number of papers published by scholars with average citations per paper, and $\mathrm{H}$-index refers to the number of $N$ papers published by a scholar, with $H$ papers being cited at least $H$ times each [12]. It not only quantifies research output but also combines the ability of researchers to publish with the impact of citation. Therefore, $\mathrm{H}$-index is the most widely used indicator among the quantitative evaluation indicators to evaluate the scientific performance of scholars. Subsequent scholars have improved $\mathrm{H}$-index on the basis of $\mathrm{H}$-index and proposed new indicators for scientific performance evaluation, such as G-index (2006) [13], R-index and AR-index (2007) [14], M-index (2008) [15], EM-index (2017) [16], and so on. However, the concept of $\mathrm{H}$-index is simple, and it is easy to calculate and has the characteristics of robustness and comprehensiveness, which makes it widely used in the evaluation of scientific performance.

2.3. Scientific Collaboration Network. The "network" is a collection of nodes and all their relationships with each other. The "scientific cooperation network" is a collection of researchers as nodes and the connections of researchers through scientific cooperation. In the scientific cooperation network, degree centrality, betweenness centrality, closeness centrality, eigenvector centrality, PageRank, and other network characteristic indicators are commonly used to describe the position, function, or influence of nodes in the network. Studies have been conducted to examine the relationship between scientific collaboration networks and scholar performance in terms of these network characteristics indicators. Abbasi et al. used G-index as the dependent variable to measure the academic influence of scholars. The network characteristics such as degree centrality, closeness centrality, betweenness centrality, eigencentrality, and average relationship strength are taken as independent variables. They studied their relation and found that the degree centricity, eigencentrality, and average relation strength are positively correlated with their academic influence (G-index). Closeness centrality and betweenness centrality are negatively correlated with their academic influence (G-index) [17]. From the perspective of social capital, Li et al. studied the relationship between degree centrality, closeness centrality, betweenness centrality of scientific cooperation network, and the average number of citations of scholars' papers. They found that degree centrality and closeness centrality had little correlation with the average number of citations, while betweenness centrality was positively correlated with the average number of citations [18]. GonzalezBrambila et al. found that having more direct contact with other scholars, being in the structural hole position of the scientific cooperation network, cooperating with scholars in other disciplines, and being in the center of the scientific cooperation network will increase the number of papers published by scholars [19]. Abbasi et al. proposed two new indicators, PDI and PTDI, on the basis of traditional social network indicators; studied the relationship between PDI and PTDI and scholars' research influence based on citation relationship; and found that PDI and PTDI were positively correlated with research influence based on citation relationship [20]. Damien et al. found that scholars who are in the bridge position of the scientific cooperation network will improve their scientific performance [21].

While these network characteristics indicators can describe to some extent the intrinsic relevance of collaboration in a research network, they do not provide a good description of the extent to which nodes in the network are connected to other important nodes. Among these commonly used network characteristic indicators, degree centrality is the most basic one. Degree centrality is a commonly used concept in social network analysis (SNA), also known as degree, and it is also the most intuitive centrality indicator in the study of scientific cooperation network. The higher the degree of a node is, the more nodes it is associated with, and the more important the node is in the network. In a cooperative network with $g$ nodes, the degree centrality of node $i$ is the total number of direct contacts between node $i$ and other $g-1$ nodes. If an author has a degree of 10 , he is associated with 10 authors in his scientific collaboration network. While degree centrality is generally used to describe the extent to which a node is connected to other nodes in a social network, it only measures the number of nodes connected to a node, not the importance of the nodes connected to a node. Therefore, Korn et al. consider a new network characteristic metric based on degree centrality to measure the degree to which a node in the network is connected to other important nodes, namely L-index (lobby index), which refers that there are at least $L$ nodes whose degrees are at least $L$ out of $N$ nodes connected to a node in the network [22]. L-index provides a new direction for the research on scientific cooperation network and scholar performance as well.

Based on this perspective, this paper explores the correlation between the degree and L-index of authorship of Physical Education and Training papers and scientific performance (number of papers, average citations per paper, and $\mathrm{H}$-index), lays a theoretical foundation for subsequent 
research related to the utility of research collaboration, provides a reference for the assessment of research performance of scholars in other disciplines, and provides a theoretical reference for more scientific and comprehensive prediction of scholars' research performance in the future.

2.4. Social Network Analysis. In the western society of the 1920 s, social network analysis was gradually applied to the fields of sociology, anthropology, psychology, and so on. Simmel first used the concept of "network" in 1922. Brown first put forward the concept of "social network" in 1940. Barnes first transformed the social network into a systematic study by analyzing the social structure of a fishing village in Norway [23]. After decades of research and exploration, social network analysis has become a unique research method, which provided a new perspective for the study of social structure. Freeman summarized the four characteristics of social network research: the analysis of the specific structure formed between the subjects of the social network, applying statistical principles and computer technology as technical support, based on data in the network, and graphic language to express social networks [24]. Wellman, an analyst of the social network, pointed out that social network was a huge network composed of social relations between groups, and social network analysis explored the deep structure under the network, specifically the connection pattern hidden under the surface of the complex social network [25]. After 2000, social network analysis has been gradually recognized and widely used in China. Bao et al. first analyzed egocentric network using the method of social network analysis [26]. Hu and Deng analyzed interpersonal relationships using the structural hole theory [27]. Liu et al. analyzed the role of small-world theory in group analysis [28]. Social network analysis is also widely used in research related to scientific collaboration. Xu and Zhu applied social network analysis to citation analysis and analyzed the influence and clique of scholars through density, centrality, and other indicators [29]. Qiu and Wang analyzed the author's cooperative relationship using social networks and found out the potential cooperative groups [30]. Xing et al. visualized the relationship between keywords by constructing the overall keyword network and combining relevant indicators of the social network, so as to more intuitively understand the research status of relevant fields [31]. Generally speaking, the social network reflects the relationship between various elements. The core of social network analysis is to analyze the "relationship" of various elements and present the relationship between individuals from "micro" to "macro" one by one. The analysis method is based on mathematical modeling and network graph, and the main research content includes the importance of each participant in the network and its role in the whole network. In addition, social network analysis and bibliometrics can also be well complementary and integrated, which are widely used in the related research of scientific cooperation network. Many scholars analyze research collaborations through bibliometric methods, but in-depth research analysis and the presentation of knowledge maps involve social network analysis methods, such as analyzing and visualizing the relationships between nodes in terms of network characteristics such as centrality, cohesive subgroups, and density, in order to identify core authors, research methods, and disciplinary hotspots in subject areas.

The scientific collaboration network consisting of the authors of our Physical Education and Training papers studied in this paper is a typical social network. Among them, each node refers to the researchers participating in the scientific cooperation, and the edge between nodes refers to the scientific cooperation between the researchers, that is, through the scientific cooperation to jointly publish the paper.

\section{Methods}

3.1. The Data Source. In 1931, Bradford, a British bibliographer, first revealed the law of literature concentration and dispersion, that is, in a certain discipline, about one-third of the literature was published in $3.2 \%$ of the periodicals. In 1967, UNESCO researched the distribution of the literature and found that $75 \%$ of the literature appeared in only $10 \%$ of the journals. In 1971, Garfield, an American intelligence scientist and scientometrician and the founder of SCI, counted the distribution of references in journals and found that $24 \%$ of the citations appeared in $1.25 \%$ of the journals. All these indicate the existence of "core effect," that is, the existence of "core journals." The input documents of core journals are authoritative and representative scientific research achievements in various disciplines after strict selection.

The data used in this paper are from core journals of sports, and the data are searched through the CSSCI database. Input, respectively, in the "journal" column the name of 16 kinds of sports core periodicals: "Journal of Beijing Sport University," "Journal of Shanghai University of Sport," "Journal of Capital University of Physical Education and Sports," "Journal of Shandong Sport University," "Journal of Chengdu Sport University," "Journal of Tianjin University of Sport," "Journal of Xi'an Institute of Physical Education," "Journal of Wuhan Institute of Physical Education," "Journal of Shenyang Sport University," "Journal of Guangzhou Sport University," "Journal of Nanjing Sport Institute (Social Science)," "China Sport Science and Technology," "Sports Culture Guide," "Journal of Physical Education," "Sports \& Science," and "China Sport Science." The subject type was set to "Physical Education," and the secondary subject type was set to "Physical Education and Training," with no restriction on publication time. Considering that the same Chinese characters will appear in the author's name, it is ambiguous to use only the name for calculation in the collected data set. In order to eliminate such ambiguity, this paper matches the name and work affiliation of the paper author at the same time, that is, two scholars with the same name and different affiliations are identified as two different scholars. Finally, the extracts saved all the literature names, authors, work affiliations of authors, 
references, publication time, citation times, and other information and finally got 10,386 data.

\subsection{Variable Selection and Measurement}

3.2.1. The Dependent Variable. The dependent variable of this study is the scientific performance of all authors in the sample, which mainly includes three aspects: the number of papers, average citations per paper, and $\mathrm{H}$-index.

(1) The Number of Papers. The number of papers refers to the total number of papers published by each author in his academic career. A certain number of papers is the basis for creating high-level scientific achievements.

(2) Average Citations per Paper. The average citations per paper are the ratio of the total number of citations per author to the number of papers. The higher an author's average citations per paper is, the higher the average quality of that author's research output and the more useful his or her results are to other scholars. The formula for calculating average citations per paper is as follows:

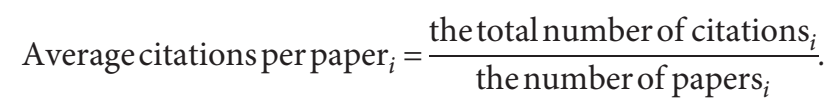

(3) $H$-Index. $H$-index refers to the number of $N$ papers published by a scholar, with $H$ papers being cited at least $H$ times each [12]. H-index can accurately reflect a person's academic achievements. H-index not only quantifies the scientific output of scholars but also takes into account the ability of researchers to publish papers and the influence of citation. The higher an author's H-index is, the more influential and academically valuable articles he or she has published.

3.2.2. The Independent Variables. The independent variables for this study are the degree centrality and L-index of all authors in the sample.

(1) Degree Centrality. Degree centrality refers to the total number of direct connections between node $i$ and other $g-$ 1 nodes in a network with $g$ nodes. The higher the degree centrality of a node is, the more nodes this node has contact with, and the more important this node is in the network. The calculation formula of degree centrality is as follows:

$$
\text { Degree centrality } y_{i}=\sum_{j=1}^{g} x_{i j} \quad(i \neq j) .
$$

(2) L-Index. L-index means the degree to which $L$ of the $N$ nodes in the network that are connected to a node is at least $L$ [22]. L-index reflects the extent to which a node in the network is connected to other important nodes. The higher L-index of an author, the more authors with high centrality he or she is associated with and the higher the quality of his or her collaborators.

\subsection{The Research Methods}

3.3.1. Establish the Scientific Cooperation Network. The scientific collaboration network in this study was constructed on the basis of our Physical Education and Training papers, which is a typical social network. In the scientific collaboration network, each node represents an author, and the edges between nodes indicate that two authors have collaborated on previous papers. The scientific collaboration network in this study is constructed by Python.

3.3.2. Measurement of the Independent and Dependent Variables. After the research collaboration network was constructed, the degree centrality and L-index of all authors in the sample were calculated using Python. Then using bibliometric methods, the research performance indicators such as the number of papers, average citations per paper, and $\mathrm{H}$-index of all authors in the sample were calculated. The distribution of all independent and dependent variables and the correlation between the independent and dependent variables were then visualized and analyzed through Python.

3.3.3. Correlation Analysis of Independent Variables and Dependent Variables. The correlation between the independent and dependent variables was analyzed using Stata to explore in detail the effects of degree centrality and L-index on scientific performance indicators such as the number of papers, average citations per paper, and $\mathrm{H}$-index.

\section{Analysis and Results}

\subsection{Descriptive Statistics and the Distribution of Independent and Dependent Variables}

4.1.1. Descriptive Statistics. By processing and analyzing all the 10,386 literature data of 16 core sports journals in China with the subject type of "Sports" and the secondary subject type of "Physical Education and Training," we obtained a total of 12,875 authors, each of whom corresponds to a node in the research collaboration network. Table 1 shows the mean, median, standard deviation, and minimum and maximum values of each variable in the research collaboration network.

As we can be seen from Table 1, the mean values of the number of papers, $\mathrm{H}$-index, degree centrality, and L-index are $1.541,1.395,2.975$, and 2.468 , respectively, and their median values are $1,1,2$, and 2 , which are very close to their minimum values $1,0,1$, and 1 , respectively, and even the median of the number of papers is consistent with the minimum values. This shows that in the research field of Physical Education and Training in China, most of the authors published fewer papers, and the levels of $\mathrm{H}$-index, degree centrality, and L-index are low. The average citation per paper is 23.848 , and the median is 14 , which is also far from the maximum citation amount of 1,268 , indicating that 
TABLE 1: The descriptive statistics of independent and dependent variables.

\begin{tabular}{|c|c|c|c|c|c|c|}
\hline Variable & $N$ & Mean & Median & Std. dev. & Min & $\operatorname{Max}$ \\
\hline The number of papers & 12,875 & 1.541 & 1 & 1.778 & 1 & 66 \\
\hline Average citation per paper & 12,875 & 23.848 & 14 & 37.333 & 0 & 1,268 \\
\hline H-index & 12,875 & 1.395 & 1 & 1.212 & 0 & 28 \\
\hline Degree & 12,875 & 2.975 & 2 & 2.93 & 1 & 83 \\
\hline L-index & 12,875 & 2.468 & 2 & 1.489 & 1 & 14 \\
\hline Career length & 12,875 & 1.927 & 1 & 2.546 & 1 & 21 \\
\hline
\end{tabular}

in the research field of Physical Education and Training in China, the citation number of papers published by most authors is relatively low.

\subsubsection{Distribution of the Independent and Dependent Variables}

\section{(1) Distribution of the Dependent Variables.}

(1) Distribution of the number of papers: Figure 1 shows the distribution of the number of papers published by the authors of Physical Education and Training in China. As can be seen from Figure 1, the overall distribution of the number of papers published by authors of Physical Education and Training in China is that the number decreases rapidly within a certain range, then tends to be stable, with the minimum value of 1 and the maximum value of 66 . The largest number of authors who published one paper is 9,768, accounting for about $77 \%$ of the total number of authors. The number of authors who published less than 5 papers is 12,447 , accounting for more than $96 \%$ of the total number of authors. The authors who published more than 10 papers are fewer than 100 . And considering that the data were collected without limiting the publication date of the paper. The earliest recorded paper was published in 2000 . In the 20 years since 2000 , the total number of published papers in this field is only 10,386, and the average annual number of published papers is only about 500. At the author level, the vast majority of authors have published a small number of papers, and only a small number of authors have a high number of publications, all of which indicate that the overall level of scientific research in the subject area of Physical Education and Training in China is still relatively limited.

(2) The distribution of average citations per paper: Figure 2 shows the distribution of average citations per paper of the authors of Physical Education and Training in China. From Figure 2, we can find that the distribution of Physical Education and Training authors in China has more authors in the lower citation distribution range, and the distribution of authors gradually decreases as the number of citations increases. Although the number of authors with high citations is relatively limited, there are also authors with high citations per article, which can also indicate that there are authors in the field of Physical Education and Training in China who have

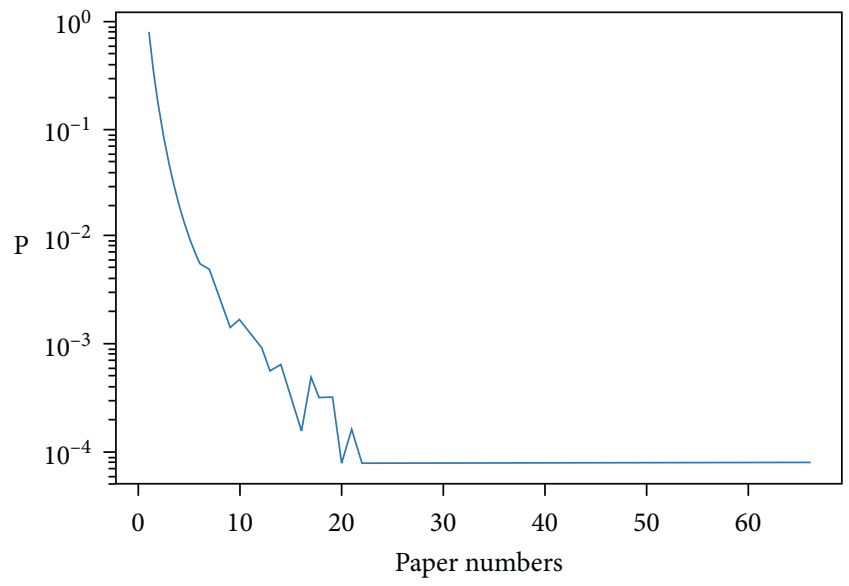

FIgURE 1: Distribution of the number of papers.

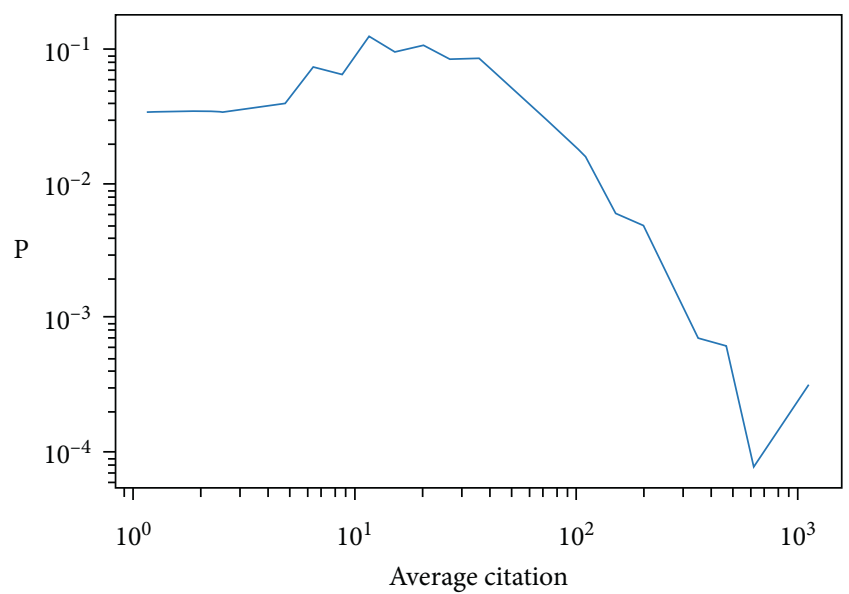

FIgURE 2: Distribution of average citations per paper.

published articles with high citations. That is, there are high impact and widely recognized scientific research results in the field of Physical Education and Training in China. However, from an overall perspective, the average number of citations per article of most authors in the field of Physical Education and Training research in China is still at a low level, and the overall level of scientific research in the discipline is still relatively limited.

(3) The distribution of H-index: Figure 3 shows the $\mathrm{H}$-index distribution of the authors of Physical Education and Training in China. From Figure 3, we can find that $\mathrm{H}$-index of Chinese Physical Education 


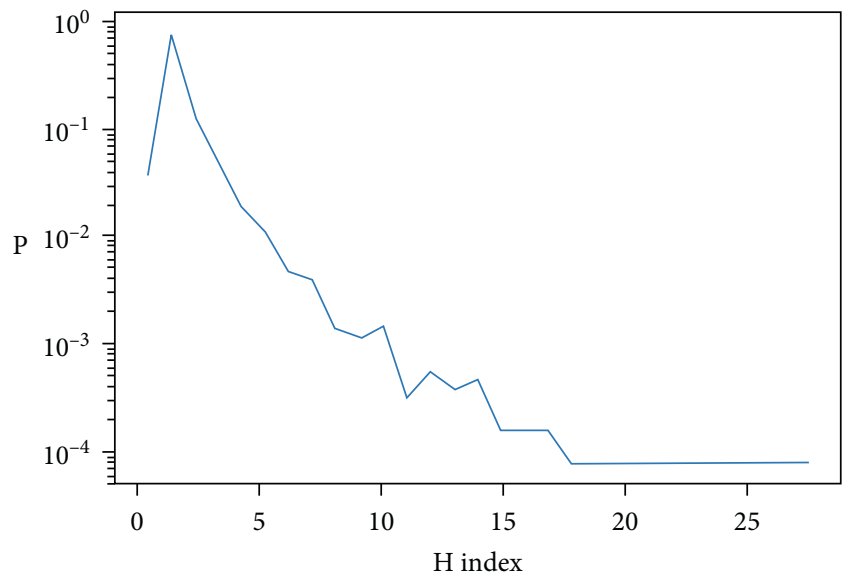

FIgURE 3: Distribution of H-index.

and Training paper authors is also on a downward trend, with a minimum value of 0 . The trend of overall distribution is that the number of authors reaches the peak when $\mathrm{H}$-index is 1 . Then, with the increase of $\mathrm{H}$-index, the number of authors decreases. When $\mathrm{H}$-index is 10 , the number of authors will reach a relatively low level. The number of authors with $\mathrm{H}$-index 1 was the largest, with a total of 9,494 , accounting for more than $73 \%$ of the total number of authors. The number of authors with a high $\mathrm{H}$-index is very small, and the number of authors with an $\mathrm{H}$-index higher than 10 is less than $1.5 \%$ of the total. It indicates that most authors have fewer published papers or the number of citations is not high, and only a few authors have a high number of published papers with a high number of citations. $\mathrm{H}$-index is the main evaluation indicator to evaluate the academic influence, so it can explain that most of the authors in the field of Physical Education and Training in China have moderate academic influence, and only a few authors have relatively higher academic influence. The scientific research influence in the field of Physical Education and Training in China needs to be improved as a whole.

(2) The Distribution of the Independent Variable.

(1) The distribution of degree centrality: Figure 4 shows the distribution of degree centrality of the authors of Chinese Physical Education and Training. From Figure 4, we can find that the degree centrality distribution of the authors of Physical Education and Training papers in China is still on a decreasing trend, and from our data, we find that the minimum value of degree centrality is 1 and the maximum value is 83 . With the increase of degree centrality, the number of authors decreases rapidly in the range of degree centrality from 1 to 10 . Since our distribution is plotted in double logarithmic coordinates, the distribution of authors slowly decreases and plateaus as the degree centrality increases when the degree centrality is greater than 10 . The authors with degree

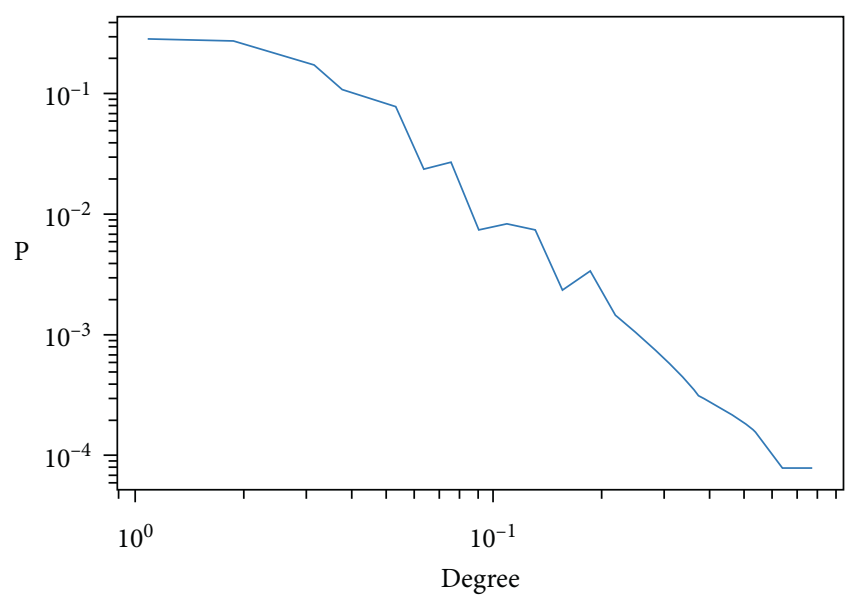

FIgURE 4: Distribution of degree centrality.

centrality 1,2 , and 3 have the largest number, with a total of 9,398 authors, accounting for more than $72 \%$ of all authors. The number of authors with degree centrality in the range of $1-10$ is 12,610 , accounting for more than $97 \%$ of the total number of authors, indicating that the degree centrality of most of the authors of Physical Education and Training in China is less than 10 , that is, the number of collaborators of most of the authors is less than 10. It can also be seen that most of the authors in the field of Physical Education and Training in China have relatively fixed collaborators, and they generally have fixed research teams, especially small teams with less than 5 members. The number of authors whose degree centrality are more than 10 is few. Even the number of authors whose degree centrality is more than 20 is less than 70. It shows that a few authors may not have a fixed team, but they will take the initiative to seek cooperation with more of the authors, or the authors in the discipline of Physical Education and Training may have the higher reputation, and there are a number of authors or research groups that will actively seek to collaborate with them.

(2) The distribution of L-index: Figure 5 shows the distribution of L-index of the authors of Physical Education and Training in China. From Figure 5, we can find that the minimum value of L-index of authors of Physical Education and Training in China is 1 , and the maximum value is 14 . The overall distribution trend is that with the increase of L-index, the number of authors decreases. When L-index is 2,1 , and 3 , the number of authors is the highest, with a total of 10,143 , accounting for more than $78 \%$ of all authors. The number of authors whose L-index was less than 6 is 12,557, accounting for more than $90 \%$ of the total number of authors. It indicates that L-index of most of the authors of Physical Education and Training in China is less than 6, that is, most of the authors do not have at least 6 cooperators whose degree centrality is greater than 6 . They have fewer collaborators or their collaborators had lower degree 


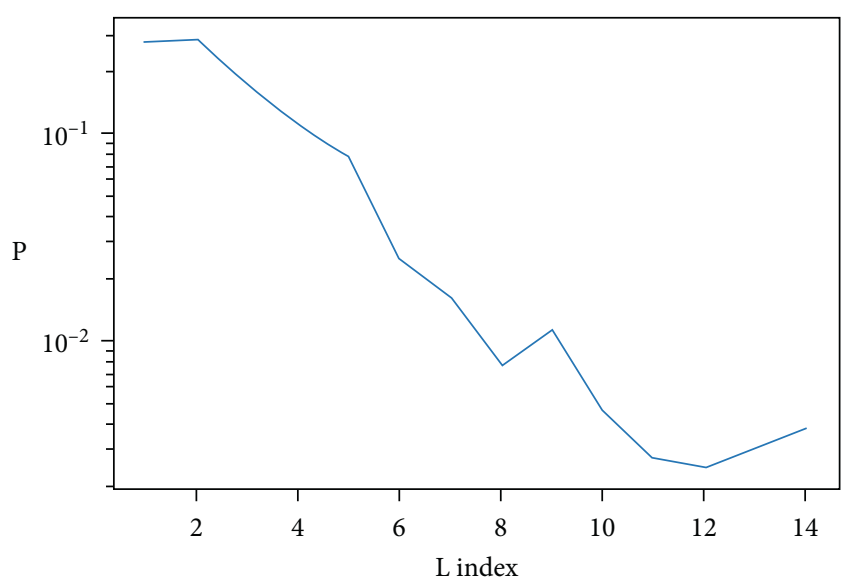

Figure 5: Distribution of L-index.

of centrality, and only a small percentage of the authors collaborate with authors who have the higher degree of centrality in the collaborative network. This shows that the authors in the field of physical education and training in China are still confined to the original small research team and can also reflect the slow development and limited research level in the field of physical education and training research in China.

4.2. Regression Analysis. The original data were initially tested for endogeneity. The explanatory variables were not correlated with the nuisance terms, and the sample size was large enough to be consistent with the "large-sample theory." This paper uses large-sample OLS regression to analyze the original data, and to avoid bias in statistical inference due to the heteroskedasticity, robust standard errors are used instead of ordinary standard errors in the large-sample OLS regression. After the regression analysis, the reasonableness of the use of large-sample OLS regression will be further tested through robustness tests.

\subsubsection{Regression Analysis of the Number of Papers and Degree} Centrality. Figure 6 shows the scatter diagram and regression curve of the correlation between the number of papers and degree centrality by Python. Each point represents an author, and the red curve represents the correlation regression curve between the number of papers and degree centrality. As can be seen from Figure 6, with the increase of degree centrality, the number of papers also increases significantly. Most of the authors are distributed in the lower-left corner of the figure, that is, most of the authors have a low degree of centrality and published a small number of papers. Then Stata is used to make unary linear regression for the number of papers and degree centrality. The results are shown in Table 2.

As can be seen from Table 2, $p$ value is less than 0.01 ; the regression coefficient is $0.347>0$, which indicates that the number of papers is positively correlated with degree centrality; and this relationship is significant at the confidence level of 0.01 . It also indicates that with the increase of

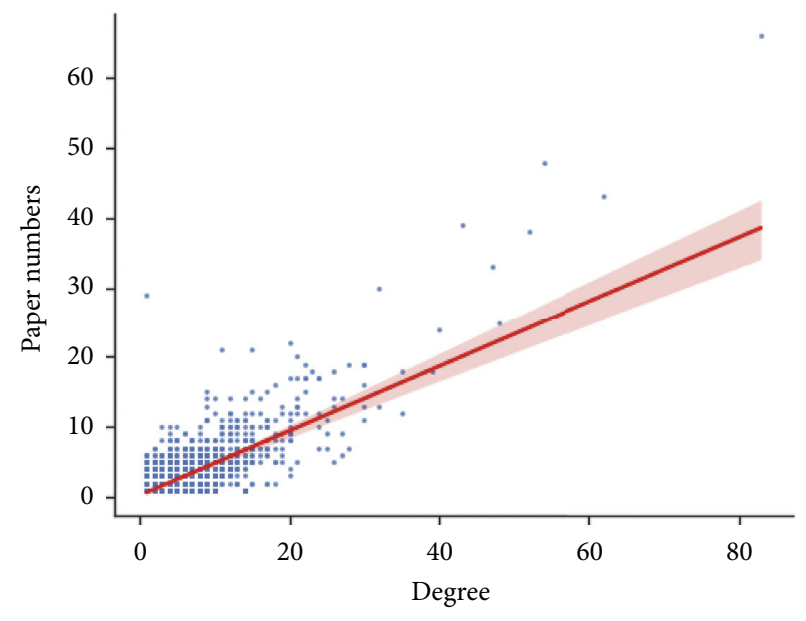

FIGURE 6: Scatter diagram and regression curve of correlation between the number of papers and degree centrality.

authors' degree centrality, the number of published papers will be higher. In other words, if an author widely seeks to cooperate with more authors, the number of published papers will be increased.

4.2.2. Regression Analysis of Average Citations per Paper and Degree Centrality. The scatter diagram and regression curve of correlation between average citations per paper and degree centrality are shown in Figure 7. Each point represents an author, and the red curve represents the correlation regression curve between average citations per paper and degree centrality. Then Stata was used to make unary linear regression for average citations per article and degree centrality. The results are shown in Table 3:

As can be seen from Table 3, $p$-value is less than 0.01; the regression coefficient is $0.484>0$, which indicates that average citations per paper are positively correlated with degree centrality; and this relationship is significant at the confidence level of 0.01 . It also indicates that with the increase of authors' degree centrality, average citations per paper will also increase, that is, if the authors seek to cooperate with more authors extensively, the average citation of his or her papers will increase.

And Figure 7 shows that the authors with higher average citations per paper have lower degree centrality, which may be due to the number of samples with low degree centrality is relatively huge, and there are some authors without high degree centrality who publish the paper with higher influence. The number of samples with high degree centrality is relatively small, and their average citations per paper have a certain gap between the authors who publish papers with high influence and whose degree centrality is not high but still higher than the average level, which will not affect the positive correlation between average citations per paper and degree centrality.

4.2.3. Regression Analysis of H-Index and Degree Centrality. The scatter diagram and regression curve of the correlation between $\mathrm{H}$-index and degree centrality are made by Python, 
TABLE 2: Unary linear regression results of the number of papers and degree centrality.

\begin{tabular}{|c|c|c|c|c|c|c|c|}
\hline The number of papers & Coeff. & Robust std. error & $t$-value & $p$-value & \multicolumn{2}{|c|}{$\begin{array}{l}\text { 95\% confidence } \\
\text { interval }\end{array}$} & Sig \\
\hline Degree & 0.347 & 0.038 & 9.17 & $\leq 0.001$ & 0.273 & 0.421 & $* * *$ \\
\hline Main journal & -0.005 & 0.002 & -2.23 & 0.026 & -0.009 & -0.001 & $* *$ \\
\hline Start year & -0.015 & 0.002 & -7.59 & $\leq 0.001$ & -0.019 & -0.011 & $* * *$ \\
\hline Career length & 0.244 & 0.017 & 14.08 & $\leq 0.001$ & 0.21 & 0.278 & $* * *$ \\
\hline Constant & 30.71 & 3.971 & 7.73 & $\leq 0.001$ & 22.927 & 38.493 & $* * *$ \\
\hline Mean dependent var & & 1.541 & & SD dependent var & & & \\
\hline$R$-squared & & 0.675 & & Number of obs & & & \\
\hline$F$-test & & 441.958 & & Prob $>F$ & & & \\
\hline Akaike crit. (AIC) & & $36,873.095$ & & Bayesian crit. (BIC) & & 36 & \\
\hline
\end{tabular}

${ }^{* * *} P<0.01,{ }^{* *} P<0.05$, and ${ }^{*} P<0.1$.

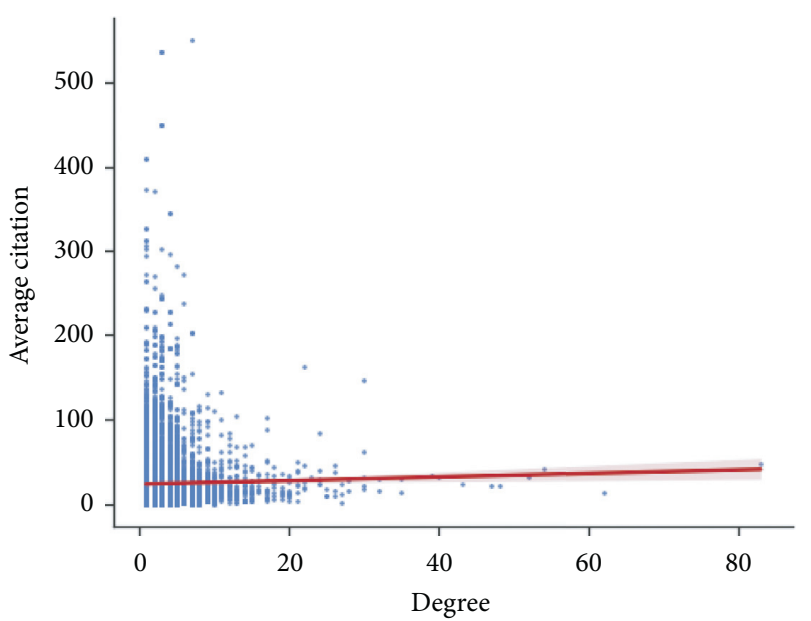

FiguRE 7: Scatter diagram and regression curve of correlation between average citations per paper and degree centrality.

TABLE 3: Unary linear regression results of average citations per paper and degree centrality.

\begin{tabular}{|c|c|c|c|c|c|c|c|}
\hline Average citations per paper & Coeff. & Robust std. error & $t$-value & $p$-value & \multicolumn{2}{|c|}{ 95\% confidence interval } & Sig \\
\hline Degree & 0.484 & 0.106 & 4.55 & $\leq 0.001$ & 0.276 & 0.693 & $* * *$ \\
\hline Main journal & -0.283 & 0.093 & -3.05 & 0.002 & -0.465 & -0.101 & $* * *$ \\
\hline Start year & -1.621 & 0.046 & -35.19 & $\leq 0.001$ & -1.711 & -1.531 & $* * *$ \\
\hline Career length & -0.754 & 0.111 & -6.78 & $\leq 0.001$ & -0.972 & -0.536 & $* * *$ \\
\hline Constant & $3,282.059$ & 92.749 & 35.39 & $\leq 0.001$ & $3,100.257$ & $3,463.86$ & $* * *$ \\
\hline Mean dependent var & & \multicolumn{2}{|l|}{23.848} & \multicolumn{2}{|c|}{ SD dependent var } & \multicolumn{2}{|c|}{37.333} \\
\hline$R$-squared & & \multicolumn{2}{|l|}{0.056} & \multicolumn{2}{|c|}{ Number of obs } & \multicolumn{2}{|c|}{12,875} \\
\hline$F$-test & & \multicolumn{2}{|l|}{358.384} & \multicolumn{2}{|c|}{ Prob $>F$} & \multicolumn{2}{|c|}{$\leq 0.001$} \\
\hline Akaike crit. (AIC) & & \multicolumn{2}{|l|}{$129,014.918$} & \multicolumn{2}{|c|}{ Bayesian crit. (BIC) } & \multicolumn{2}{|c|}{$129,052.233$} \\
\hline
\end{tabular}

${ }^{* * *} P<0.01,{ }^{* *} P<0.05$, and ${ }^{*} P<0.1$.

as shown in Figure 8. Each point represents an author, and the red curve represents the correlation regression curve between $\mathrm{H}$-index and degree centrality. As can be seen from Figure 8, with the increase of degree centrality, $\mathrm{H}$-index also increases significantly. Most of the authors are distributed in the lower-left corner of the figure, that is, most of the authors have low degree centrality and low $\mathrm{H}$-index. Then Stata was used to make unary linear regression of $\mathrm{H}$-index and degree centrality. The results are shown in Table 4:

As can be seen from Table 4, $p$-value is less than 0.01 ; the regression coefficient is $0.188>0$, which indicates that $\mathrm{H}$-index is positively correlated with degree centrality; and this relationship is significant at the confidence level of 0.01 . It also indicates that with the increase of degree centrality, the quantity and quality of published articles will also be higher. In other word, it also indicates that if an author widely seeks to collaborate with more authors, the quantity and quality of his published articles will be significantly increased, that is, his or her academic influence will be significantly increased.

4.2.4. Regression Analysis of the Number of Papers and L-Index. The scatter diagram and regression curve of the correlation between the number of papers and L-index are 


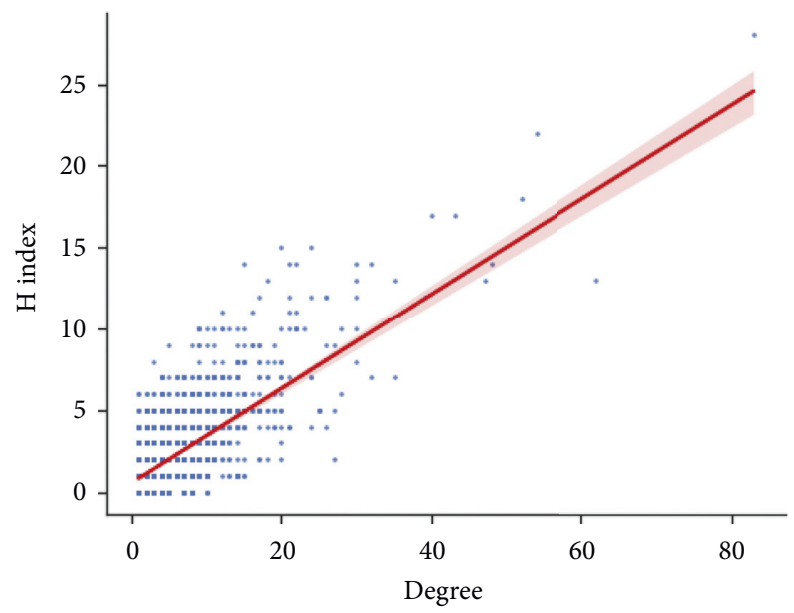

FiguRE 8: Scatter diagram and regression curve of correlation between H-index and degree centrality.

TABLE 4: Unary linear regression results of $\mathrm{H}$-index and degree centrality.

\begin{tabular}{|c|c|c|c|c|c|c|c|}
\hline \multirow{2}{*}{$\begin{array}{l}\text { H-index } \\
\text { Degree }\end{array}$} & \multirow{2}{*}{$\begin{array}{c}\text { Coeff. } \\
0.188\end{array}$} & \multirow{2}{*}{$\begin{array}{c}\text { Robust std. error } \\
0.011\end{array}$} & \multirow{2}{*}{$\frac{t \text {-value }}{16.47}$} & \multirow{2}{*}{$\frac{p \text {-value }}{\leq 0.001}$} & \multicolumn{2}{|c|}{$\begin{array}{l}\text { 95\% confidence } \\
\text { interval }\end{array}$} & \multirow{2}{*}{$\frac{\mathrm{Si}}{* *}$} \\
\hline & & & & & 0.166 & 0.211 & \\
\hline Main journal & -0.01 & 0.002 & -6.16 & $\leq 0.001$ & -0.013 & -0.007 & \\
\hline Start year & -0.021 & 0.001 & -18.78 & $\leq 0.001$ & -0.023 & -0.019 & \\
\hline Career length & 0.213 & 0.008 & 25.34 & $\leq 0.001$ & 0.196 & 0.229 & \\
\hline Constant & 42.232 & 2.215 & 19.07 & $\leq 0.001$ & 37.89 & 46.574 & \\
\hline Mean dependent var & & 1.395 & & SD dependent var & & & \\
\hline$R$-squared & & 0.659 & & Number of obs & & & \\
\hline$F$-test & & 612.121 & & Prob $>F$ & & & \\
\hline Akaike crit. (AIC) & & $27,629.154$ & & Bayesian crit. (BIC) & & 27 & \\
\hline
\end{tabular}

${ }^{* * *} P<0.01,{ }^{* *} P<0.05$, and ${ }^{*} P<0.1$.

made by Python, as shown in Figure 9. Each point represents an author, and the red curve represents the regression curve of correlation between the number of papers and L-index. As can be seen from Figure 9, with the increase of L-index, the number of papers also increases significantly. Most of the authors are distributed in the range where L-index is less than 10. When L-index is greater than 10 , the distribution of the number of papers fluctuates due to the decrease of the sample size, but it does not affect the overall trend. Then Stata was used to make unary linear regression of the number of papers and L-index. The results are shown in Table 5.

As can be seen from Table 5, $p$ value is less than 0.01 ; regression coefficient is $0.153>0$, which indicates that there is a positive correlation between the number of papers and L-index; and this relationship is significant at the confidence level of 0.01 . It also indicates that with the increase of the author's L-index, the number of published articles will be higher. In other words, if more authors seek to cooperate with more important and influential authors in the research collaboration network, that is, the more authors seek to cooperate with a higher L-index, the number of published papers will increase.

4.2.5. Regression Analysis of Average Citations per Paper and $L$-Index. The scatter diagram and regression curve of correlation between average citations per paper and L-index are

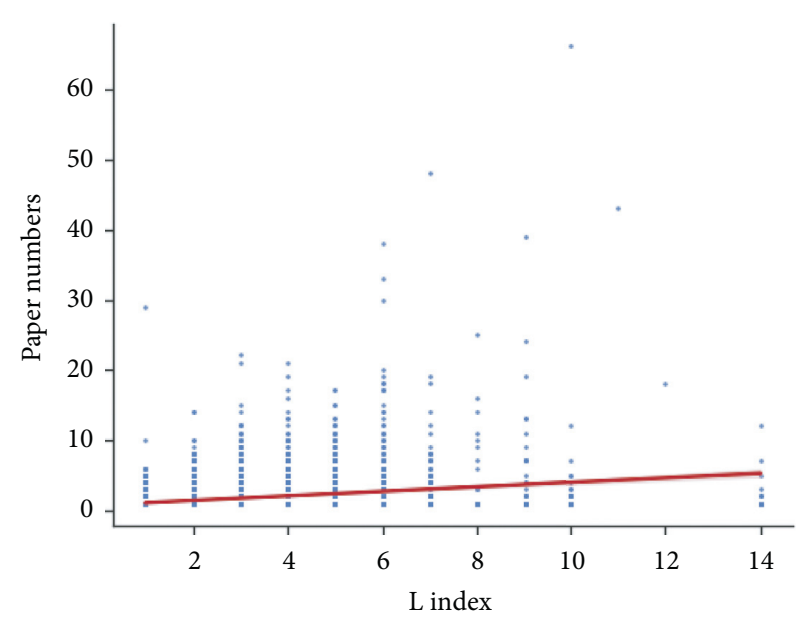

FIGURE 9: Scatter diagram and regression curve of correlation between the number of papers and L-index.

shown in Figure 10. Each point represents an author, and the red curve represents the correlation regression curve between average citations per paper and L-index. As can be seen from Figure 10, with the increase of L-index, the maximum of the average citations per paper decreases. According to the correlation regression curve between average citations per paper and L-index, average citations per paper and L-index do not show an obvious correlation. Then 
TABLE 5: Unary linear regression results of the number of papers and L-index.

\begin{tabular}{|c|c|c|c|c|c|c|c|}
\hline The number of papers & Coeff. & Robust std. error & $t$-value & $p$-value & \multicolumn{2}{|c|}{$\begin{array}{c}\text { 95\% confidence } \\
\text { interval }\end{array}$} & Sig \\
\hline L-index & 0.153 & 0.019 & 7.96 & $\leq 0.001$ & 0.115 & 0.191 & $* * *$ \\
\hline Main journal & -0.01 & 0.003 & -3.62 & $\leq 0.001$ & -0.015 & -0.004 & $* * *$ \\
\hline Start year & -0.005 & 0.001 & -3.98 & $\leq 0.001$ & -0.008 & -0.003 & $* * *$ \\
\hline Career length & 0.442 & 0.02 & 22.26 & $\leq 0.001$ & 0.403 & 0.481 & $* * *$ \\
\hline Constant & 11.229 & 2.71 & 4.14 & $\leq 0.001$ & 5.918 & 16.54 & $* * *$ \\
\hline Mean dependent var & & 1.541 & & SD dependent var & & & \\
\hline$R$-squared & & 0.461 & & Number of obs & & & \\
\hline$F$-test & & 152.352 & & Prob $>F$ & & & \\
\hline Akaike crit. (AIC) & & $43,397.556$ & & Bayesian crit. (BIC) & & 43,4 & \\
\hline
\end{tabular}

${ }^{* * *} P<0.01,{ }^{* *} P<0.05$, and ${ }^{*} P<0.1$.

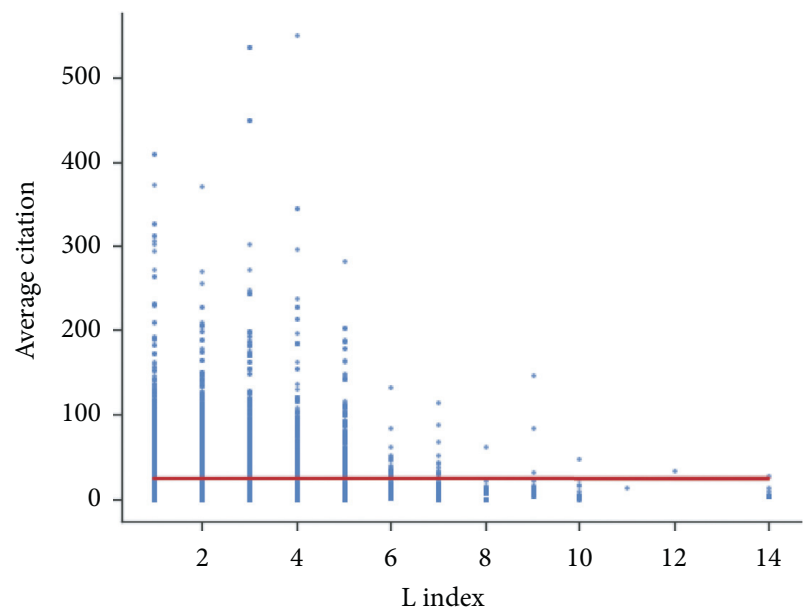

FIGURE 10: Scatter diagram and regression curve of correlation between average citations per paper and L-index.

Stata was used to make unary linear regression between average citations per paper and L-index. The results are shown in Table 6.

As can be seen from Table $6, p$-value is less than 0.01 ; the regression coefficient is $0.926>0$, which indicates that there is a positive correlation between average citations per paper and L-index; and this relationship is significant at the confidence level of 0.01 . It also indicates that with the increase of the author's L-index, the average citations per paper will be higher. In other words, if more authors seek to cooperate with more important and influential authors in the collaboration network, that is, the more authors seek to cooperate with a higher L-index, average citations per paper will increase.

4.2.6. Regression Analysis of H-Index and L-Index. The scatter diagram and regression curve of correlation between $\mathrm{H}$-index and L-index are shown in Figure 11. Each point represents an author, and the red curve represents the regression curve of correlation between $\mathrm{H}$-index and L-index. As can be seen from Figure 11, with the increase of L-index, $\mathrm{H}$-index also increases. Then Stata was used to make unary linear regression of $\mathrm{H}$-index and $\mathrm{L}$-index. The results are shown in Table 7:
As can be seen from Table 7 , the $p$-value is less than 0.01 ; the regression coefficient is $0.095>0$, which indicates that $\mathrm{H}$-index is positively correlated with L-index; and this relationship is significant at the confidence level of 0.01 . It also means that as the author's L-index goes up, his or her $\mathrm{H}$-index also goes up. In other words, if more authors seek to cooperate with authors with higher L-index, that is, more authors seek to cooperate with more important and more influential authors in the scientific research collaboration network, the quantity and quality of their published papers will be improved.

4.3. Robustness Test. In reality, the research performance of the author will be affected by other variables except some of the author's own characteristics in the cooperation network, so his research performance is not a result of random allocation. In order to reduce the influence of other factors such as selection bias, this study uses the method of propensity score matching (PSM) to estimate the influence of degree centrality and L-index on the research performance of paper authors. When evaluating the research performance of the authors, we find a control group that is as similar as possible to the treatment group through the propensity score value and conduct a paired analysis; then the sample selection bias can be effectively reduced; and the observable factors such as control variables can be effectively removed. The author's research performance is affected, and the average treatment effect (ATT) after eliminating the selection bias can be obtained. Therefore, after selecting the most published journals, the starting year of the author's career, and the length of the author's career as matching variables, the PSM method can better avoid the general regression analysis of the degree centrality and the L-index on the research performance of the author. The estimated error in the past can effectively solve the endogenous problem. In addition, this study uses the nearest neighbor matching method to match the propensity value. This method can achieve the "one" to "all" (i.e., individuals in each treatment group and individuals in all control groups) matching and can also avoid degree centrality and L-index and the twoway causal effect of the author's research performance. The results of propensity score matching are shown in Table 8.

According to the empirical results in Table 8, taking the influence of degree centrality on the number of papers as an 
TABLE 6: Unary linear regression results of average citations per paper and L-index.

\begin{tabular}{|c|c|c|c|c|c|c|c|}
\hline Average citations per paper & Coeff. & Robust std. error & $t$-value & $p$-value & \multicolumn{2}{|c|}{ 95\% confidence interval } & Sig \\
\hline L-index & 0.926 & 0.216 & 4.29 & $\leq 0.001$ & 0.503 & 1.35 & $* * *$ \\
\hline Main journal & -0.288 & 0.093 & -3.11 & 0.002 & -0.47 & -0.107 & $* * *$ \\
\hline Start year & -1.635 & 0.047 & -34.70 & $\leq 0.001$ & -1.728 & -1.543 & $* * *$ \\
\hline Career length & -0.584 & 0.095 & -6.16 & $\leq 0.001$ & -0.769 & -0.398 & $* * *$ \\
\hline Constant & $3,309.679$ & 94.786 & 34.92 & $\leq 0.001$ & $3,123.884$ & $3,495.475$ & $* * *$ \\
\hline Mean dependent var & & 23.848 & \multicolumn{3}{|c|}{ SD dependent var } & \multicolumn{2}{|c|}{37.333} \\
\hline$R$-squared & & 0.056 & \multicolumn{3}{|c|}{ Number of obs } & \multicolumn{2}{|c|}{12,875} \\
\hline$F$-test & & 358.430 & \multicolumn{3}{|c|}{ Prob $>F$} & \multicolumn{2}{|c|}{$\leq 0.001$} \\
\hline Akaike crit. (AIC) & & $129,011.476$ & \multicolumn{3}{|c|}{ Bayesian crit. (BIC) } & \multicolumn{2}{|c|}{$129,048.791$} \\
\hline
\end{tabular}

${ }^{* * *} p<0.01,{ }^{* *} p<0.05$, and ${ }^{*} p<0.1$.

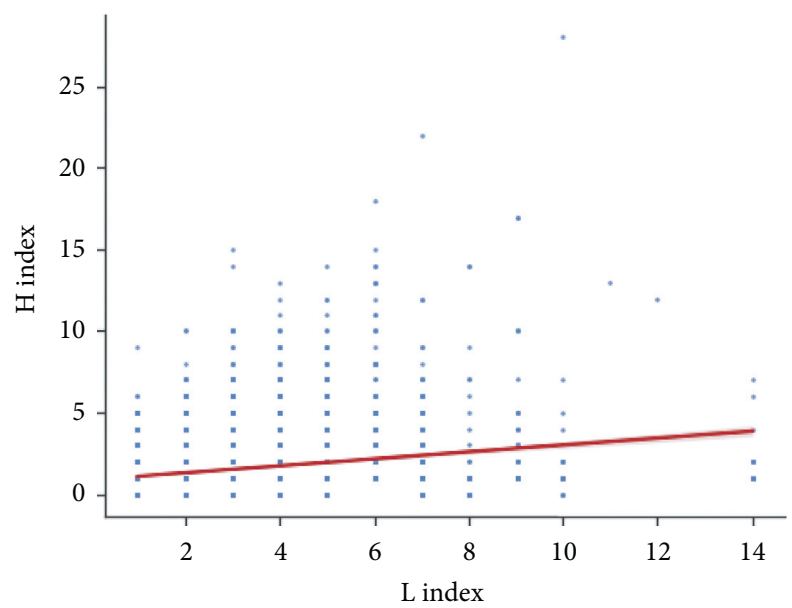

FIGURE 11: Scatter diagram and regression curve of correlation between $\mathrm{H}$-index and L-index.

TABLE 7: Unary linear regression results of $\mathrm{H}$-index and L-index.

\begin{tabular}{|c|c|c|c|c|c|c|c|}
\hline H-index & Coeff. & Robust std. error & $t$-value & $p$-value & \multicolumn{2}{|c|}{$\begin{array}{l}\text { 95\% confidence } \\
\text { interval }\end{array}$} & Sig \\
\hline L-index & 0.095 & 0.009 & 10.45 & $\leq 0.001$ & 0.077 & 0.113 & $* * *$ \\
\hline Main journal & -0.012 & 0.002 & -6.71 & $\leq 0.001$ & -0.016 & -0.009 & $* * *$ \\
\hline Start year & -0.016 & 0.001 & -14.94 & $\leq 0.001$ & -0.018 & -0.014 & $* * *$ \\
\hline Career length & 0.319 & 0.01 & 31.11 & $\leq 0.001$ & 0.298 & 0.339 & $* * *$ \\
\hline Constant & 32.61 & 2.137 & 15.26 & $\leq 0.001$ & 28.421 & 36.799 & $* * *$ \\
\hline Mean dependent var & & 1.395 & & SD dependent var & & & \\
\hline$R$-squared & & 0.527 & & Number of obs & & & \\
\hline$F$-test & & 326.115 & & Prob $>F$ & & & \\
\hline Akaike crit. (AIC) & & $31,861.910$ & & Bayesian crit. (BIC) & & 31 , & \\
\hline
\end{tabular}

${ }^{* * *} p<0.01,{ }^{* *} p<0.05,{ }^{*} p<0.1$.

example, after matching, the average number of papers in the treatment group is 2.360; the average number of papers in the control group is 1.707; and the average treatment effect of ATT is 0.652 show great significance at $1 \%$ level. The test based on the PSM method shows that after controlling for the influence of other factors, the number of papers for authors with a higher level of degree centrality is 0.652 on average higher than that of authors with a lower level of degree centrality, which is about $38 \%$, which means degree centrality. There is a significant improvement effect on the number of papers. In the same way, the L-index has a significant effect on the number of papers, the degree centrality of the papers is cited, and the degree centrality and the L-index have a significant effect on the $\mathrm{H}$-index.

In order to verify the sample characteristics of the main variables before and after the matching and the balance of the matching, the balance test was carried out according to different independent variables. The test results are shown in Tables 9 and 10. The standard deviation of each matching variable after the matching is small. According to Rosenbaum and Rubin's point of view, the absolute value of the standard deviation value of the variable after the matching is significantly less than 20 , which can be considered the 
TABle 8: Propensity score matching results.

\begin{tabular}{|c|c|c|c|c|c|c|}
\hline Dependent variable & Scientific performance & Treatment group & Control group & ATT & Standard error & $T$-value \\
\hline Degree centrality & \multirow{2}{*}{ The number of papers } & 2.360 & 1.707 & 0.652 & 0.048 & $13.35^{* * *}$ \\
\hline L-index & & 2.185 & 1.742 & 0.442 & 0.063 & $6.96^{* * *}$ \\
\hline Degree centrality & \multirow{2}{*}{ Average citations per article } & 25.061 & 22.344 & 2.717 & 1.130 & $2.40^{* * *}$ \\
\hline L-index & & - & - & - & - & - \\
\hline Degree centrality & \multirow{2}{*}{$\mathrm{H}$-index } & 2.044 & 1.577 & 0.467 & 0.034 & $13.62^{* * *}$ \\
\hline L-index & & 1.850 & 1.553 & 0.297 & 0.040 & $7.39^{* * *}$ \\
\hline
\end{tabular}

TABle 9: Degree centrality balance test.

\begin{tabular}{|c|c|c|c|c|c|c|c|c|}
\hline \multirow{2}{*}{ Variable } & \multirow{2}{*}{ Unmatched/matched } & \multicolumn{2}{|c|}{ Mean } & \multicolumn{2}{|c|}{$\%$ reduction } & \multicolumn{2}{|c|}{$t$-test } & \multirow{2}{*}{$V(T) / V(C)$} \\
\hline & & Treated & Control & $\%$ bias & |bias| & $t$ & $p>|t|$ & \\
\hline \multirow{2}{*}{ Main journal } & Unmatched & 4.7676 & 5.0682 & -8.0 & & -4.01 & 0.000 & $0.92^{*}$ \\
\hline & Matched & 4.7887 & 4.7817 & 0.2 & 97.7 & 0.08 & 0.937 & 0.99 \\
\hline \multirow{2}{*}{ Start year } & Unmatched & 2009 & 2008.9 & 3.0 & & 1.54 & 0.124 & $1.12^{*}$ \\
\hline & Matched & 2009.1 & 2009.1 & -0.3 & 90.5 & -0.12 & 0.907 & 1.02 \\
\hline \multirow{2}{*}{ Career length } & Unmatched & 3.3256 & 1.4093 & 64.9 & & 40.24 & 0.000 & $6.59^{*}$ \\
\hline & Matched & 3.1639 & 3.1619 & 0.1 & 99.9 & 0.02 & 0.981 & 1.00 \\
\hline
\end{tabular}

TABLE 10: L-index balance test.

\begin{tabular}{|c|c|c|c|c|c|c|c|c|}
\hline \multirow{2}{*}{ Variable } & \multirow{2}{*}{ Unmatched/matched } & \multicolumn{2}{|c|}{ Mean } & \multicolumn{2}{|c|}{$\%$ reduction } & \multicolumn{2}{|c|}{$t$-test } & \multirow{2}{*}{$V(T) / V(C)$} \\
\hline & & Treated & Control & $\%$ bias & |bias & $t$ & $p>|t|$ & \\
\hline \multirow{2}{*}{ Main journal } & Unmatched & 5.0425 & 4.9721 & 1.9 & & 0.86 & 0.388 & $0.92^{*}$ \\
\hline & Matched & 5.0469 & 4.9897 & 1.5 & 18.8 & 0.57 & 0.565 & 0.99 \\
\hline \multirow{2}{*}{ Start year } & Unmatched & 2009.5 & 2008.8 & 12.6 & & 5.99 & 0.000 & $1.18 *$ \\
\hline & Matched & 2009.5 & 2009.5 & 0.2 & 98.7 & 0.06 & 0.954 & 1.00 \\
\hline \multirow{2}{*}{ Career length } & Unmatched & 2.7577 & 1.703 & 35.4 & & 19.50 & 0.000 & $3.04^{*}$ \\
\hline & Matched & 2.7376 & 2.7461 & -0.3 & 99.2 & -0.09 & 0.931 & 0.99 \\
\hline
\end{tabular}

matching method is suitable and the effect is better. Therefore, the propensity score matching result this time is more reliable.

The results of the robustness tests indicate that there is no endogeneity problem and also validate the rationality of using large-sample OLS regressions.

\section{Research Conclusions and Implications}

In this study, the correlation between the degree centrality and L-index of authors and scientific performance (number of papers, average citations per paper, and $\mathrm{H}$-index) was analyzed in 16 core journals of Physical Education and Training, a second category discipline under the first category Sports, and the specific findings are as follows: (1) degree centrality is positively correlated with the number of papers, average citation per paper, and H-index and (2) L-index is positively correlated with the number of papers, average citations per paper, and $\mathrm{H}$-index. From the results of this study, it can be seen that if the authors seek to cooperate with more authors extensively, especially with more influential authors, their research performance will be improved.

This study also has some theoretical contributions. This article analyzes the correlation between degree centrality, L-index of the authors in Physical Education and Training, and scientific performance. It lays a theoretical foundation for subsequent researches on the effectiveness of scientific cooperation, provides a theoretical reference for predicting the scientific performance of scholars in a more scientific and comprehensive way in the future, and also provides a reference for the evaluation of the scientific performance of scholars in other disciplines.

In addition, this study also has some shortcomings. First of all, there is no in-depth study on the reasons why there is no correlation between average citations per paper and L-index. Second, the data of this study are 16 core journals of Physical Education and Training science in China, which define the subject area, and the research results may not be extrapolated to other fields. These need to be further explored in the follow-up research.

\section{Data Availability}

The data are available at the Chinese Social Sciences Citation Index (CSSCI) database (http://cssrac.nju.edu.cn/).

\section{Conflicts of Interest}

The authors declare that there are no conflicts of interest.

\section{Authors' Contributions}

Bin Zhang wrote the original draft, reviewed and edited the manuscript, and contributed literature collection. Jian $\mathrm{Wu}$ determined the framework of the paper, was responsible for 
funding acquisition, and reviewed and edited the manuscript. Qian Huang wrote the original draft. Yujiao Tan and Lu Zhang reviewed and edited the manuscript. Qian Zheng, Yu Zhang, and Miao He contributed to the literature collection. Wei Wang was responsible for funding acquisition.

\section{Acknowledgments}

This work was supported in part by the National Social Science Foundation of China under Grant no. 20ATY007.

\section{References}

[1] D. J. S. Price, "Little science, big science," Von Der Studierstube Zur, vol. 7, no. 3-6, pp. 443-458, 1963.

[2] A. G. Heffner, "Funded research, multiple authorship, and subauthorship collaboration in four disciplines," Scientometrics, vol. 3, no. 1, pp. 5-12, 1981.

[3] J. Ziman and R. W. Schmitt, "Prometheus bound: science in a dynamic steady state," American Journal of Physics, vol. 63, no. 5, pp. 476-477, 1995.

[4] J. S. Katz and B. R. Martin, "What is research collaboration?" Research Policy, vol. 26, no. 1, pp. 1-18, 1997.

[5] R. Zhao and F. Wen, "Scientific research collaboration and knowledge communication," Library and Information Service, vol. 55, no. 20, pp. 6-27, 2011.

[6] Y. Okubo, Bibliometric Indicators and Analysis of Research Systems: Methods and ExamplesOECD Publishing, Paris, France, 1997.

[7] Wuyishan and L. Liang, "Some problems that should be paid attention to in quantitative evaluation of scientific research performance by using bibliometrics indicators," Chinese Journal of Scientific and Technical Periodicals, vol. 2, pp. 110-111, 2001.

[8] Y. Zhang, "The influence factors should be used correctly to evaluate sci-tech periodicals and papers," Acta Editologica, vol. 4, pp. 214-215, 1998.

[9] Wuyishan, "Some situations about SCI as a tool for scientific research performance evaluation," Chinese Journal of Scientific and Technical Periodicals, vol. 1, pp. 39-41, 2002.

[10] J. Pang, An. Scientific Metrological Research Methodology, Scientific and Technical Documentation Press, Beijing, China, 1999.

[11] G. Yang, Metric Analysis on Scientific Papers and Evaluation of Scientific Performance for Military Medical University, PLA Air Force Military Medical University, Xi'an, China, 2003.

[12] J. E. Hirsch, “An index to quantify an individual's scientific research output," Proceedings of the National Academy of Sciences of the United States of America, vol. 102, no. 46, 2005.

[13] L. Egghe, "Theory and practise of the g-index," Scientometrics, vol. 69, no. 1, pp. 131-152, 2006.

[14] J. Bihui, L. Liming, R. Rousseau, and L. Egghe, “The R- and AR-indices: complementing the h-index[J]," Chinese Science Bulletin, vol. 52, no. 6, pp. 855-863, 2007.

[15] L. Bornmann and H. D. Daniel, Are There Better Indices for Evaluation Purposes than the H Index? A Comparison of Nine Different Variants of the H Index Using Data from Biomedicine, John Wiley \& Sons, Inc., Hoboken, NJ, USA, 2008.

[16] B. Anand and S. Tripathi, "EM-index: a new measure to evaluate the scientific impact of scientists," Scientometrics, vol. 112, no. 1, pp. 659-677, 2017.

[17] A. Abbasi, J. Altmann, and L. Hossain, "Identifying the effects of co-authorship networks on the performance of scholars: a correlation and regression analysis of performance measures and social network analysis measures," Journal of Informetrics, vol. 5, no. 4, pp. 594-607, 2011.

[18] E. Y. Li, C. H. Liao, and H. R. Yen, "Co-authorship networks and research impact: a social capital perspective," Research Policy, vol. 42, no. 9, 2013.

[19] C. N. Gonzalez-Brambila, F. M. Veloso, and D. Krackhardt, "The impact of network embeddedness on research output," Research Policy, vol. 42, no. 9, 2013.

[20] A. Abbasi, R. T. Wigand, and L. Hossain, "Measuring social capital through network analysis and its influence on individual performance," Library \& Information Science Research, vol. 36, no. 1, pp. 66-73, 2014.

[21] C. Damien, D. Arnaud, L. Catherine, and M. Perroux, "The impact of a researcher's structural position on scientific performance: an empirical analysis," PLoS One, vol. 11, no. 8, Article ID e0161281, 2016.

[22] A. Korn, A. Schubert, and A. Telcs, "Lobby index in networks," Physica A: Statistical Mechanics and Its Applications, vol. 388, no. 11, pp. 2221-2226, 2009.

[23] J. A. Barnes, Class and Committees in a Norwegian Island Parish, 1954.

[24] L. C. Freeman, "Centrality in social networks: conceptual clarification,” Social Networks, vol. 1, no. 3, pp. 215-239, 1979.

[25] A. Herz, A. Müller, and B. Wellman, "Structural analysis: from method and metaphor to theory and substance," in Social Structures: A Network Approach, B. Wellman and S. D. Berkowitz, Eds., Cambridge University Press, Cambridge, UK, 2019.

[26] C. Bao, X. Xie, and N. Shen, "Analysis of interpersonal networks," Journal of The China Society for Scientific and Technical Information, vol. 22, no. 3, pp. 365-374, 2003.

[27] R. Hu and X. Deng, "Research on personal interpersonal network analysis system based on structure hole theory," Journal of The China Society for Scientific and Technical Information, vol. 24, no. 4, pp. 485-489, 2005.

[28] Z. Liu, L. Yin, and D. Xu, "The application of complex network analysis method in collaborative research," Science and Technology Management Research, vol. 25, no. 12, pp. 267269, 2005.

[29] Y. Xu and Q. Zhu, "Demonstration study of social network analysis method in citation analysis," Information Studies: Theory \& Application, vol. 2, pp. 184-188, 2008.

[30] J. Qiu and F. Wang, "Analysis on author cooperation relationship of competitive intelligence research in China based on SNA," Library Tribune, vol. 30, no. 6, pp. 34-134, 2010.

[31] J. Xing, W. Dong, D. Jia, and L. Yao, "Discussion on the study of digital library based on keywords network analysis," $L i$ brary Work and Study, vol. 8, pp. 35-38, 2011. 